\title{
Learning Analytics como ferramenta para a análise do desempenho dos alunos em Cursos Semipresenciais
}

\author{
Wesley Reuel M. Silva ${ }^{1}$, Jéssica Laísa D. da Silva ${ }^{1}$, \\ Fabiano R. Ugulino ${ }^{1}$, Marcia Lucena ${ }^{1}$, Isabel D. Nunes ${ }^{1}$ \\ ${ }^{1}$ Instituto Metrópole Digital - Universidade Federal do Rio Grande do Norte (UFRN) \\ Av. Sen. Salgado Filho 3000 - 59.078-970 - Natal - RN - Brasil \\ \{wreuel,jessicalaisajl, ugulino.fabiano\}@gmail.com, marciaj@dimap.ufrn.br, \\ beleimd.ufrn.br
}

\begin{abstract}
The blended teaching comes to confirm as an education form where the students know the Distance Learning but still have the necessary classroom support. The evalution in this modality should therefore consider the two forms of action, both classroom activities and the acess and utilization of virtual environment should be relevant. This article is intend to identify how acess to the virtual environment can influence the student performance, both virutally and face-to-face, from a case study in the Blended Course Technical of Digital Metropolis Institute of UFRN and raise questions to improve course which follow the same model.
\end{abstract}

Resumo. $O$ ensino semipresencial vem-se confirmando como uma forma de educação em que os alunos conhecem o ensino a distância mas possuem o apoio presencial necessário. A avaliação nessa modalidade deve-se, portanto, considerar as duas formas de atuação, tanto atividades presenciais quanto o acesso e a utilização do ambiente virtual devem ser relevantes. Este artigo tem o objetivo de identificar como o acesso ao ambiente virtual pode influenciar no desempenho dos alunos, tanto virtualmente quanto presencialmente, a partir de um estudo de caso no Curso Técnico Semipresencial do Instituto Metrópole Digital (IMD) da UFRN e levantar questões para o melhoramento em cursos que seguem essa modalidade.

\section{Introdução}

A modalidade de cursos semipresenciais ou híbridos utiliza recursos didáticos (material instrucional, livros-texto e ambiente virtual de aprendizagem) que permite ao estudante organizar seus horários de estudo conforme sua disponibilidade de tempo e local. Tais características são atrativas para o aumento da procura por esses cursos. Conforme o Censo da Associação Brasileira de Educação a Distância (ABED) no ano de 2014, constam a soma de 3.453 cursos regulamentados semipresenciais ou disciplinas EaD (ABED, 2014, p. 43).

Assim, os gestores e educadores de cursos híbridos devem ter a preocupação de promover uma parceria entre o Ambiente Virtual de Aprendizagem (AVA) e suas atividades com os recursos e tarefas realizadas presencialmente. Essa parceria deve ter 
V Congresso Brasileiro de Informática na Educação (CBIE 2016)

Anais do XXVII Simpósio Brasileiro de Informática na Educação (SBIE 2016)

como objetivo o melhoramento da aprendizagem, motivando o aluno a praticar a distância como apoio ao ensino presencial (Ribeiro et al., 2014).

O trabalho de Corbellini e Real (2012) investiga uma disciplina semipresencial numa Universidade Pública. Este trabalho analisa a forma como os alunos se apropriam dos ambientes de aprendizagem no espaço físico (sala de aula convencional) e no virtual e como resultado mostram que o estímulo à cooperação e ao trabalho em grupo no ambiente virtual, bem como, a prática concomitante das modalidades presencial e virtual facilitam o desenvolvimento do processo de aprendizagem.

Já o artigo de Tori (2002) estuda o conceito de distância nas atividades de aprendizagem e propõe uma métrica para facilitar suas comparações. São identificados três tipos de distâncias na educação (espacial, temporal e interativa) e três tipos de interações (aluno-professor, aluno-aluno e aluno-material). A combinação entre elas gera 512 possibilidades de distanciamento, ou aproximação, em atividades de aprendizagem. Como resultado o artigo apresenta uma fórmula para cálculo de um parâmetro comparativo dos potenciais de proximidade das atividades de aprendizagem e sugere a convergência da educação a distância e da presencial.

Uma forma de avaliar se as duas vertentes (presencial e virtual) estão colaborando entre si é por meio das técnicas de Learning Analytics (LA). LA visa avaliar os dados gerados a partir da execução de cursos, como participação em ambientes virtuais de aprendizagem, frequência e notas para identificar situações como o andamento da aprendizagem e comportamento dos alunos com o intuito de melhorar o ensino (Santos et al. 2014).

Este trabalho tem como objetivo utilizar técnicas de Learning Analytics para analisar a situação de 807 alunos do Curso Técnico Semipresencial de Tecnologia da Informação do Instituto Metrópole Digital (IMD) da Universidade Federal do Rio Grande do Norte (UFRN) referente à contribuição da utilização do ambiente virtual em colaboração às atividades presenciais. Os resultados encontrados permitem identificar um padrão de comportamento entre os alunos e assim fornecer uma base para a proposta de novos formatos de acompanhamento virtual do aluno.

Este artigo encontra-se estruturado em seis seções. A Seção 2 mostra uma visão geral sobre os Cursos Semipresenciais. A Seção 3 descreve Learning Analytics e suas características. A Seção 4 apresenta a metodologia do estudo de caso trabalhado. A Seção 5 mostra os resultados analisados e por fim, as considerações finais e trabalhos futuros.

\section{Educação Híbrida ou Semipresencial}

O número de cursos semipresenciais está aumentando desde 2000 (Ribeiro et al., 2014), possibilitando também o aumento do acesso à educação juntamente com cursos unicamente on-line. Pode-se caracterizar a modalidade semipresencial, também chamada de educação híbrida ou flexível, como um contexto educacional que combina atividades a distância com o ensino presencial (Camillo, 2011). Propiciando assim, uma melhorara no desempenho dos alunos tanto no ensino presencial quanto no ensino a distância

Bahia et al. (2007) ressalta algumas vantagens em cursos semipresenciais, são elas: (i) Acompanhamento individual e permanente dos alunos, principalmente em turmas que 
V Congresso Brasileiro de Informática na Educação (CBIE 2016)

Anais do XXVII Simpósio Brasileiro de Informática na Educação (SBIE 2016)

possuem um grande número de discentes; (ii) Melhoramento na organização do tempo das atividades, já que devem ser entregues em datas marcadas e com a restrição de um Ambiente Virtual de Aprendizagem; (iii) Flexibilização do tempo e espaço do aluno; (iv) Além do contato presencial, as opiniões e avaliações são registradas e feedbacks podem ser constantemente acessados, como também acesso à opinião dos colegas;(v) Melhoramento na qualidade do material e sua disponibilização; (vi) Encorajamento para a colaboração e comunicação no Ambiente Virtual de Aprendizagem, principalmente para alunos mais tímidos que não o fariam presencialmente.

Porém, ao mesmo tempo que Bahia et al. (2007) ressalta como uma das vantagens de um curso semipresencial, Ribeiro et al. (2014) relata em seu trabalho que a dificuldade em adaptação ao método pode ser um problema, pois o aluno precisa entender como organizar seu tempo para priorizar o que deve estudar e acessar no Ambiente Virtual de Aprendizagem com intuito de auxiliar nas atividades presenciais. Assim, vantagens que são descritas para cursos desta natureza são funcionais se realmente os alunos se adaptarem ao método.

Além disso, um desafio ressaltado por Camillo (2011) é combinar e coordenar o componente presencial com o a distância, fazendo com que se complementem e se apóiem entre si e não dificultando a interação e comunicação entre alunos e professores.

\section{Learning Analytics}

A proposta de Learning Analytics - LA é identificar, por meio da extração e análise de dados de forma automática, perfis de alunos, problemas ou padrões com um determinado conteúdo programático, reconhecimento entre o sucesso e insucesso de trajetórias de aprendizagem (designs instrucionais), detecção de lições complexas, entre outras (Jovanovic et al., 2008).

Reforçando esse conceito, no $1^{\circ}$ Internacional Conference on Learning Analytics and Knowledge - LAK (LAK, 2011) estabeleceu Learning Analytics como a medição, coleta, análise e disponibilização de dados sobre alunos e seus contextos, com o propósito de entender e aperfeiçoar a aprendizagem e o ambiente onde ocorre (Ferguson, 2012).

O processo de LA possui cinco passos (Santos, 2014), incluindo as fases de melhoramento do curso, que são: Capturar, Reportar, Predizer, Adaptar, Personalizar e Intervir. As fases de Capturar e Reportar estão ligadas ao acesso aos dados, sua organização e extração. Predizer, permite que técnicas de LA sejam aplicadas para o entendimento dos dados obtidos a partir de um problema. Por fim, as três últimas fases, Adaptar, Personalisar e Intervir, estabelecem meios para o melhoramento do curso e assim aumentar o desempenho dos alunos.

As técnicas utilizadas para realização de Learning Analytics podem ser: Análise de Redes Sociais (em que são analisadas a conexão entre alunos e professores, dependendo de sua frequência, qualidade ou importância) (Shum and Ferguson, 2011); Processamento da Linguagem Natural (a análise léxica, sintática e semântica de um texto pode mostrar o nível de interesse e aprofundamento de um aluno em um determinado assunto); Predição (análise de dados atuais e históricos para prever eventos futuros) (Siemens, 2012); Determinação de Risco (identificação de alunos em risco a partir de sua perfomance no curso) (Arnold, 
V Congresso Brasileiro de Informática na Educação (CBIE 2016)

Anais do XXVII Simpósio Brasileiro de Informática na Educação (SBIE 2016)

2010); Sequenciamento de curso (permite disponibilizar sequenciamentos diferentes para cada aluno conforme seu perfil) (Brusilovsky and Vassileva, 2003) e Identificação de alunos que precisam de ajuda (identifica quem, professores ou outros alunos, que podem ajudá-lo em suas tarefas) (Siemens, 2012).

\section{Estudo de Caso}

O estudo de caso foi realizado no Instituto Metrópole Digital (IMD) da UFRN. O IMD oferece a cada ano em média 1.680 vagas de alunos para o Curso Técnico Semipresencial de Tecnologia da Informação (TI) visando à qualificação profissional na área. Nos momentos virtuais a interação ocorre por meio do Ambiente Virtual de Aprendizagem Modular Object-Oriented Dynamic Learning Environment (MOODLE) (Moran, 2009). Já as aulas presenciais são realizadas nos pólos do IMD (nas cidades de Natal, Caicó, Mossoró e Angicos do Rio Grande do Norte), tendo um horário semanal fixo para cada turma.

\subsection{Metodologia}

A metodologia utilizada neste trabalho é uma pesquisa quantitativa na forma de um estudo de caso do Módulo Básico do Curso Técnico Semipresencial para o semestre 2015.1 do pólo da cidade de Natal.

A metodologia deste trabalho é baseado no processo de Learning Analytics que segue os seguinte passos: Capturar dados, Reportar dados, Predizer, Adaptar, Personalisar e Intervir. Este artigo tem o foco nas fases de captação e divulgação das informações obtidas. A predição, adaptação, personlização e intervenção são os próximos passos a partir da análise realizada. As quatro (4) fases realizadas foram: Formulação do problema, Identificação dos dados, Extração e Visualização dos resultados e Análise dos resultados. Cada fase será apresentada a seguir.

1. Formulação do problema:

A questão de problema identificada é: As duas vertentes do ensino semipresencial, virtual e presencial, estão contribuindo entre si para o melhoramento do desempenho dos alunos?

2. Identificação dos dados

O Curso Técnico está dividido em três módulos: Básico, Intermediário e Avançado. Nesta pesquisa foram utilizados somente os dados do Módulo Básico por ser o primeiro contato dos alunos com o ensino na modalidade semipresencial. Os dados utilizados foram do semestre 2015.1, o qual contém 6 disciplinas (Arquitetura de Computadores, Inglês Técnico I, Introdução às Tecnologias da Informação, Lógica de Programação, Matemática Aplicada e Sistemas Operacionais). Os alunos do Módulo Básico estão divididos em 20 turmas (nomeadas de T01 a T20) totalizando 807 alunos. A Figura 1 mostra o número de alunos por turma. 
V Congresso Brasileiro de Informática na Educação (CBIE 2016)

Anais do XXVII Simpósio Brasileiro de Informática na Educação (SBIE 2016)

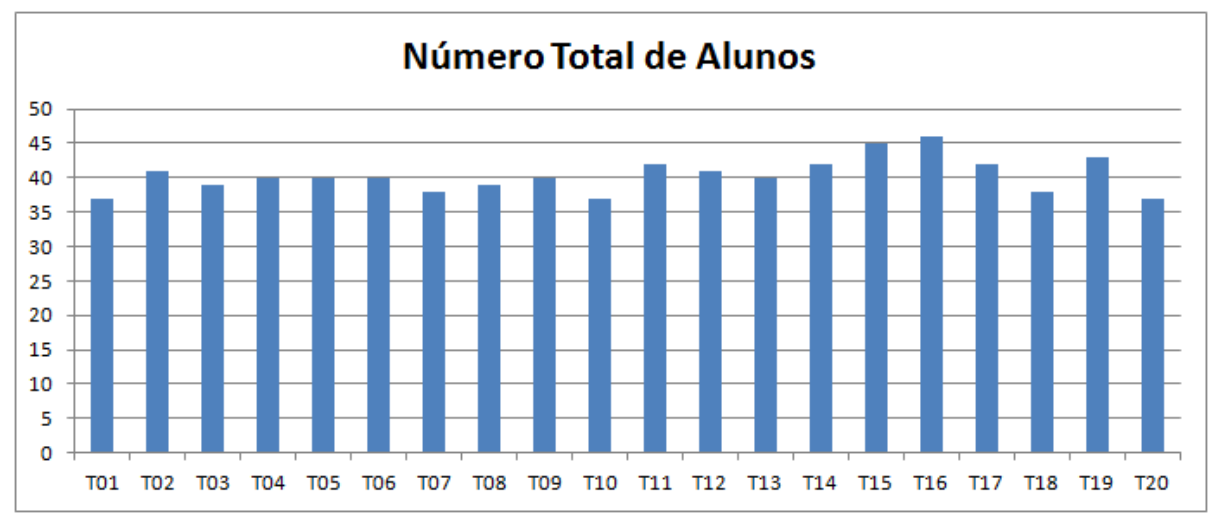

Figura 1. Número de alunos por turma do Nível Básico do Curso Técnico em 2015.1

Os dados foram obtidos por meio de dois sistemas utilizados no Curso Técnico: o Ambiente Virtual de Aprendizagem Moodle e o Sistema Integrado de Gestão de Atividades Acadêmicas (SIGAA). O Moodle é utilizado para prover o apoio para as atividades à distância, contendo tarefas, interação e comunicação por meio de fóruns e chats e a organização das disciplinas de cada módulo. O SIGAA permite o armazenamento de notas e frequências das atividades realizadas, tanto das atividades realizadas presencialmente quanto das atividades desenvolvidas por meio do ambiente virtual.

Os dados do SIGAA foram: nota de Participação Virtual (PV), nota de Participação Presencial (PP) e status do aluno (aprovado ou reprovado). A nota PV engloba a frequência do aluno no ambiente virtual e a presença em atividades como fórum, mensagens e chats. Já a nota PP é fornecida pelo tutor seguindo os seguintes critérios: assiduidade, comprimento dos prazos de entrega das atividades, resolução de exercícios em sala de aula e interesse nas atividades (Gomes et al., 2012).

As notas PV e PP são utilizadas para definir a nota de Participação na Turma (PT). Segundo Gomes et al. (2012) a nota PV deveria ter um peso de $70 \%$ da nota, para $30 \%$ da nota de participação presencial. Porém, observou-se nos dados obtidos que o cálculo realizado era a média simples, com peso igual de $50 \%$ para cada nota.

A média final do aluno é calculada por meio da fórmula, onde AE são Atividades Executadas e PE é a nota da Prova Escrita:

$$
N F=\frac{P T+A E+(P E \times 2)}{4}
$$

Neste trabalho somente foram consideradas as notas PP e PV devido à questão de pesquisa, onde o foco é considerar como a participação virtual influencia no desempenho do aluno em relação à participação presencial.

Outra informação relevante para o estudo foi o número de acessos realizados por cada aluno, conforme os critérios considerados para o cálculo da nota de Participação Virtual (acesso ao ambiente e às atividades). Essa informação foi obtida por meio do log de execução de cada aluno participante, sendo contabilizados o número total de acessos. 
V Congresso Brasileiro de Informática na Educação (CBIE 2016)

Anais do XXVII Simpósio Brasileiro de Informática na Educação (SBIE 2016)

\section{Extração e Visualização dos resultados}

Os dados foram obtidos por meio de consultas ao Banco de Dados dos respectivos ambientes (Moodle e SIGAA). As informações pertinentes para responder à questão de pesquisa são visualizadas por meio de gráficos de barras e de dispersão. $\mathrm{O}$ gráfico de dispersão é necessário devido à necessidade de analisar duas informações com granularidades diferentes como também por mostrar uma tendência na correlação entre as informações.

4. Análise dos resultados

Após a terceira fase, os dados foram analisados a partir de gráficos gerados conforme a questão de pesquisa. Tais resultados podem ser visualizados na próxima seção deste artigo.

\section{Resultados e Análise dos Resultados}

O foco do trabalho é identificar se as duas vertentes da aprendizagem semipresencial estão contribuindo entre si para o melhoramento do aprendizado do aluno. A partir da extração do dados, observou-se que as turmas são ecléticas no sentido de números de aprovados e reprovados. O gráfico da Figura 2 mostra que metade das turmas tiveram o número de aprovados maior do que de reprovados, e o contrário com o restante das turmas. Tal cenário é interessante para avaliar como ocorre a participação virtual e presencial dos alunos já que tem-se diversidade entre as turmas.

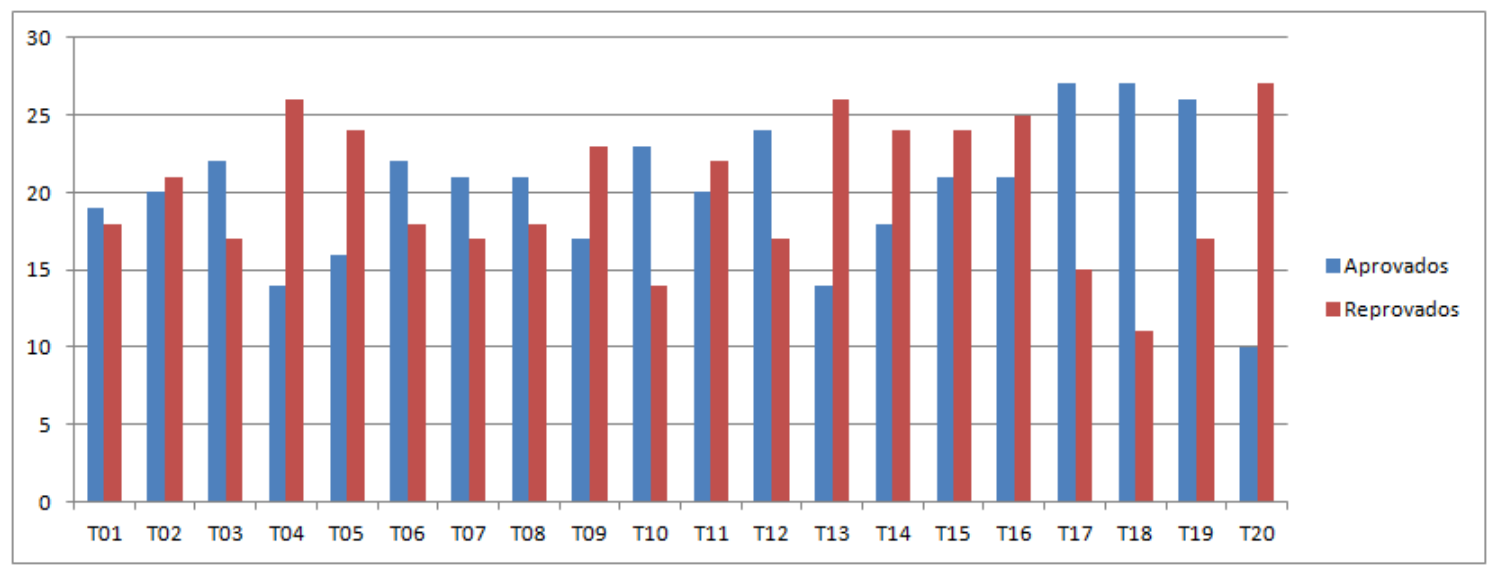

Figura 2. Número de alunos aprovados e reprovados por turma

Assim, para ressaltar se o acesso possui alguma relação com as notas foi gerado os gráficos da Figura 3 e Figura 4. A Figura 3 mostra a relação do aumento do número de acessos com o aumento da nota da Participação Virtual, enquanto a Figura 4 faz a relação dos acessos com a Participação Presencial (nesse ponto, a relação de acessos com a nota PP não fica tão clara).

As notas de participação virtual e presencial são utilizadas para compor a média de Participação Total que será parte integrante da nota final do semestre do aluno. A influência do uso do ambiente virtual em tais notas fica mais evidente principalmente quando é analisado a relação de acessos com a média de Participação Total. A Figura 5 mostra essa relação para as 20 turmas analisadas. 
V Congresso Brasileiro de Informática na Educação (CBIE 2016)

Anais do XXVII Simpósio Brasileiro de Informática na Educação (SBIE 2016)

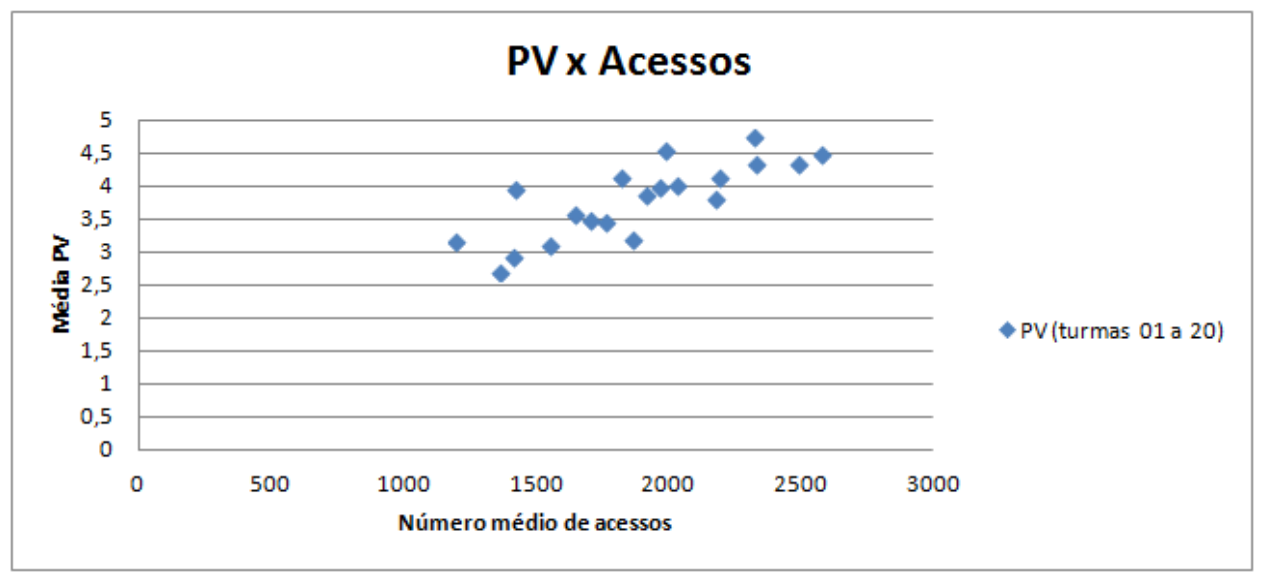

Figura 3. Nota da PV em relação ao número de acessos para todas as turmas

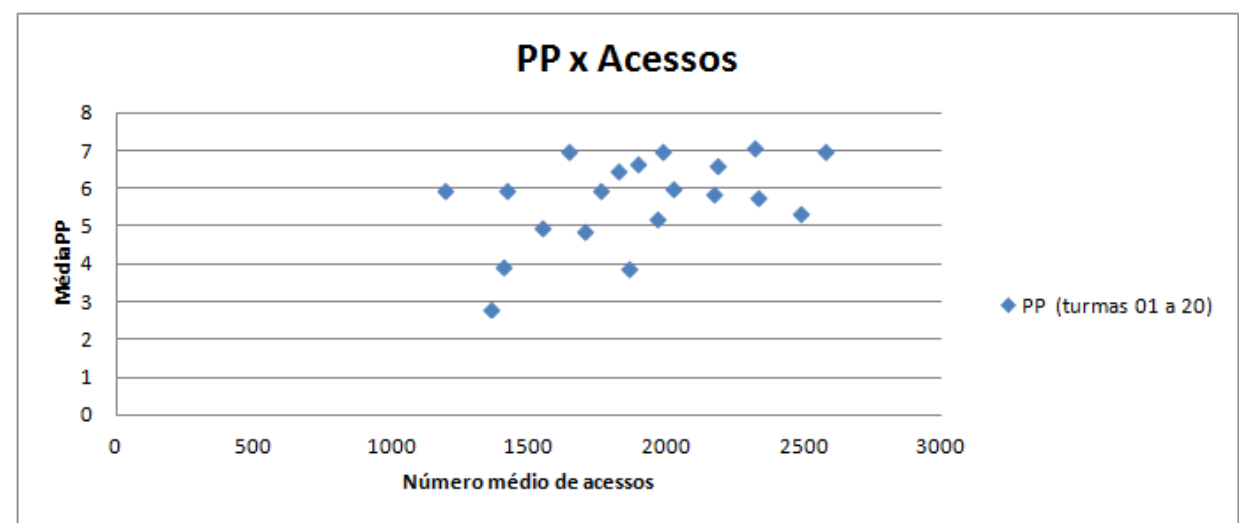

Figura 4. Nota da PP em relação ao número de acessos para todas as turmas

Como o intuito é analisar a relação entre a participação virtual e a participação presencial dos alunos, é necessário obter as notas médias de cada tipo de participação separadamente, o que pode ser visualizado na Figura 6. É possível observar que mesmo com o perfil diferente das turmas em relação ao número de aprovados e reprovados, e a tendência de correlação entre número de acessos e nota da Participação Presencial e Participação Virtual, a nota da Participação Virtual sempre é mais baixa que a nota da Participação Presencial.

Os gráficos das Figuras 5 e 6 mostram como os alunos que possuem maior participação no ambiente virtual aumentam suas notas (tanto a nota presencial como a virtual), mas não fica claro o motivo da nota virtual ser sempre mais baixa que a nota presencial. Deve-se então levar em consideração como a nota PV é contabilizada, pois não considera uma verdadeira participação no ambiente virtual, mas somente o acesso a esse ambiente.

Assim as notas obtidas de PV e PP levantam uma preocupação de que os alunos não estão utilizando e aproveitando exatamente o ambiente virtual para seu aprendizado, pois 
V Congresso Brasileiro de Informática na Educação (CBIE 2016)

Anais do XXVII Simpósio Brasileiro de Informática na Educação (SBIE 2016)

suas notas não acompanham as notas da participação presencial. A nota PV considera somente o acesso ao ambiente e não realmente como o aluno participa e qual o grau de sua participação, se sua contribuição em um fórum é realmente relevante ou não, por exemplo.

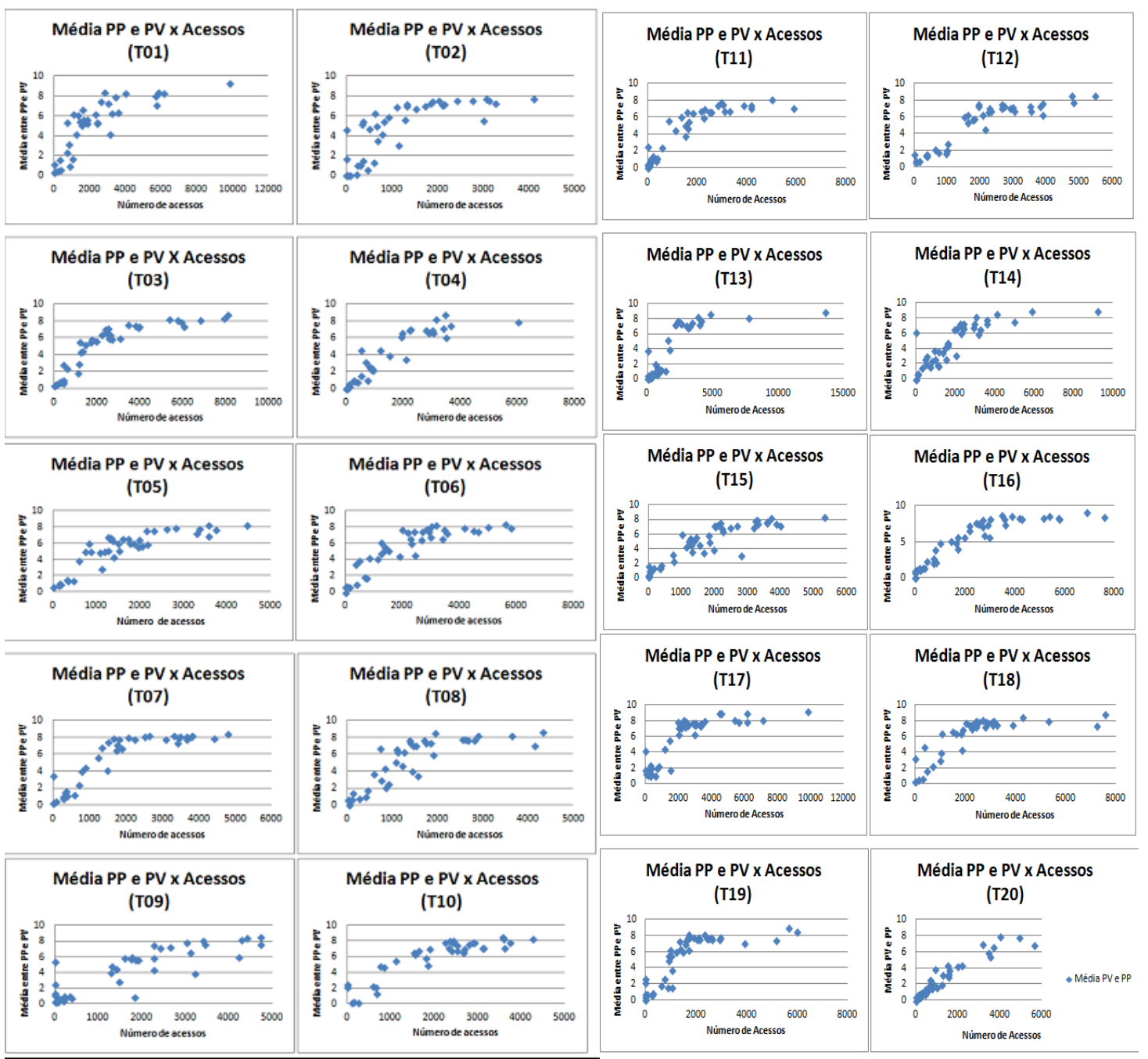

Figura 5. Média de Participação Virtual e Presencial por número de Acessos

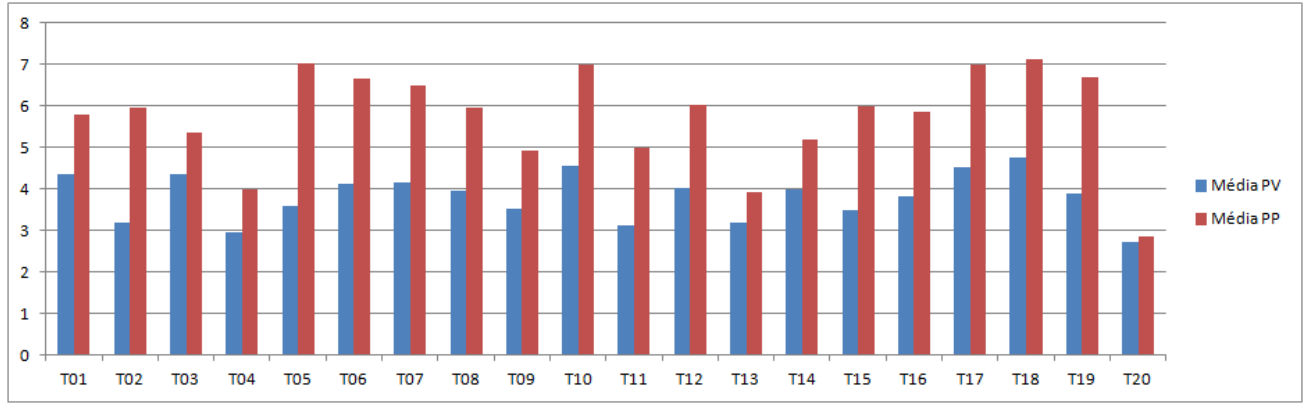

Figura 6. Média da nota de Participação Virtual e Presencial de cada turma 
V Congresso Brasileiro de Informática na Educação (CBIE 2016)

Anais do XXVII Simpósio Brasileiro de Informática na Educação (SBIE 2016)

A partir da análise realizada deve-se repensar em como a participação do aluno no ambiente virtual deve ser considerada. Segundo Silva e Silva (2007), a avaliação em ambientes virtuais segue processos metodológicos definidos a partir de objetivos propostos, considerando a participação colaborativa e autônoma do aluno. Atividades de colaboração como análise textual da participação do aluno em fóruns, chats e mensagens e construção de atividades em grupo, como também a análise de intervenção do aluno nas atividades e solução de problemas.

\section{Conclusões}

A avaliação de alunos em ambientes semipresenciais torna-se uma preocupação maior quando é considerado que as duas vertentes de ensino devem ser complementares e colaborarem entre si. Identificar se essa relação realmente está acontecendo após um período de execução de um determinado curso e, se necessário, aplicar melhorias para aumentar o desempenho dos alunos é papel da área de Learning Analytics. Essa área permite identificar quais os problemas que podem estar acontecendo e que não estejam tão explícitos e assim permitir que intervenções sejam realizadas.

Com o intuito de identificar situações de problema entre atividades presenciais e a distância em cursos semipresenciais, foi utilizado como estudo de caso o Curso Técnico Semipresencial de Tecnologia da Informação do Instituto Metrópole Digital da UFRN. Os dados obtidos mostram que o acesso ao ambiente virtual do curso apoia tanto o ensino presencial quanto o a distância. Porém, o desempenho do aluno no ambiente virtual, por meio de suas atividades e colaborações, não é considerado, prejudicando sua avaliação virtual. Tal resultado torna-se contraditório, pois mostra como é importante que o aluno se motive virtualmente para que estabeleça um desempenho melhor e como tais atuações não são consideradas.

A partir dos resultados encontrados, vê-se a necessidade de obter maiores detalhes sobre a relação entre os alunos e o ambiente virtual, mostrando, por exemplo, se os acessos realizados ao ambiente são meros acessos aos materiais e como eles interagem com outros alunos, professores e tutores. Além disso, pretende-se propor uma ferramenta automatizada que obtenha tais informações e proponha soluções de avaliação em cursos semipresenciais que tenham como suporte o AVA Moodle.

\section{Referências Bibliográficas}

ABED (2014) “Censo EAD”. Associação Brasileira de Educação a Distância (ABED Org.). São Paulo: Pearson Education do Brasil.

Arnold, K. E. (2010) “Signals: Applying Academic Analytics” Educause Quartely, volume 33, number 1 .

Bahia, N. P.; Perrotti, E. M. B.; Gomes, M. P. da S.; Pitombeira, C. V. (2007) "Disciplinas Semipresenciais e Cursos de Graduação a Distância: Avaliação da Implantação. $13^{\circ}$ Congresso Internacional de Educação a Distância. Curitiba-PR.

Brusilovsky, P.; Vassileva, J. (2003) "Course sequencing techniques for large-scale web-based education” Int. J. Cont. Engineering Education and Lifelong Learning, Vol. 13, Nos.1/2. 
V Congresso Brasileiro de Informática na Educação (CBIE 2016)

Anais do XXVII Simpósio Brasileiro de Informática na Educação (SBIE 2016)

Camillo, R. D. (2011) "Interação no componente on-line de cursos semipresenciais: a visão dos participantes” In: Linguagens e Diálogos, v. 2, n. 2, p. 45-68.

Coberllini, S. e Real, L. C. (2012) "Educação semipresencial: espaços e tempos complementares?" Anais do $23^{\circ}$ SBIE.

Ferguson, R. (2012) “The State Of Learning Analytics in 2012: A Review and Future Challenges" Technical Report KMI-12-01, Knowledge Media Institute, The Open University, UK. http://kmi.open.ac.uk/publications/techreport/kmi-12-01.

Gomes, A. V.; Oliveira, D. S. da S.; Medeiros, A. da C. (2012) "GENOME: uma ferramenta avaliativa de participação vitual" Workshop sobre avaliação e Acompanhamento da Aprendizagem em Ambientes Virtuais do CBIE - Congresso Brasileiro de Informática na Educação.

Jovanovic, J.; Gasevic, D.; Brooks, C.; Devedzic, V.; Hatala, M.; Eap, T.; Richards, G. (2008) "LOCO-Analyst: semantic web technologies in learning content usage analysis" Int. J. Cont. Engineering Education and Lifelong Learning, Vol. 18, No 1. Pag. 54- 76.

LAK (2011) "1st International Conference on Learning Analytics and Knowledge" Disponível em: https://tekri.athabascau.ca/analytics/.

Moran J, M. (2009) "Modelos e Avaliação do Ensino Superior a Distância no Brasil" ETD - Educação Temática Digital, Campinas, v.10, n.2, p.54-70.

Ribeiro, G. O.; Pinto, F. A. P.; Silva, T. E. V.; Vasconcelos, F. H. L.; Nunes, A. O. "Perspectivas para a Redução da Evasão em EaD a partir da Avaliação da Qualidade do Ensino Online" 20a Workshop de Informática na Escola (WIE 2014) In 3o Congresso Brasileiro de Informática na Educação (CBIE 2014).

Santos, A. G. dos; Nunes, I. D.; Schiel, U. (2014) “Aplicação de Learning Analytics ao Design Instrucional” XXV Simpósio Brasileiro de Informática na Educação (SBIE) In 3o Congresso Brasileiro de Informática na Educação (CBIE 2014).

Shum, S. B and Ferguson, R. (2011) "Social Learning Analytics" Tchnical Report KMI11-01, Knowledge Media Institute, The Open Univertity, UK. Disponível em: http://kmi.open.ac.uk/publications/pdf/kmi-11-01.pdf.

Siemens, G. (2012) "Sensemaking: beyond Analytics as technical activity" EDUCAUSE - Learning Initiative.

Silva, L. e Silva, M. (2007) "A Avaliação online num Ambiente Virtual de Aprendizagem" V E-TIC Encontro de Educação e Tecnologias de Informação e Comunicação.

Tori, R. (2002) "Métricas para uma Educação sem Distância." Revista Brasileira de Informática na Educação, SBC, v. 10, n. 2. 